\title{
Patient Experiences with Avelumab in Treatment-Naïve Metastatic Merkel Cell Carcinoma: Longitudinal Qualitative Interview Findings from JAVELIN Merkel 200, a Registrational Clinical Trial
}

\author{
Jérémy Lambert ${ }^{1}$ - Alexia Marrel ${ }^{1}$. Sandra P. D'Angelo ${ }^{2} \cdot$ Melissa A. Burgess ${ }^{3}$ - Bartosz Chmielowski ${ }^{4}$ - Nicola Fazio ${ }^{5}$. \\ Thilo Gambichler ${ }^{6}$. Jean-Jacques Grob ${ }^{7}$. Céleste Lebbé ${ }^{8}$. Caroline Robert ${ }^{9} \cdot$ Jeffrey Russell $^{10}$. Gülseren Güzel ${ }^{11}$. \\ Murtuza Bharmal ${ }^{12}$
}

Published online: 30 May 2020

(c) The Author(s) 2020

\begin{abstract}
Background and Objective Avelumab is approved for the treatment of metastatic Merkel cell carcinoma, a rare aggressive skin cancer with a poor prognosis. The aim of this qualitative study embedded in a clinical trial was to explore patient experiences while receiving avelumab.

Methods All treatment-naïve patients with metastatic Merkel cell carcinoma entering part B of the phase II, open-label, international, JAVELIN Merkel 200 trial (NCT02155647) were invited to participate in optional semi-structured phone interviews before avelumab administration (baseline) and at weeks 13 and 25. Interviews were conducted by trained professionals, audio-recorded, transcribed and analysed. Key concepts identified at baseline were assessed during follow-up interviews.

Results Twenty-nine patients completed the baseline interview; 19 had at least one follow-up interview. Baseline interviews described the patients' challenging journeys before being correctly diagnosed with Merkel cell carcinoma, the negative psychological burden of living with a symptomless disease and the hope for avelumab to be a successful therapy. During the trial, most patients reported an increased or continued sense of hope and willingness to fight metastatic Merkel cell carcinoma. Patients who self-reported disease improvement $(n=12)$ also reported stability or improvement in physical well-being and ability to do daily activities, having more energy, worrying less and being optimistic. Six patients who reported their condition as stable $(n=4)$ or worsened $(n=3)$ reported a worsening of physical well-being. Nine patients reported fatigue/ tiredness on the day of and after receiving avelumab. Baseline and longitudinal experiences were similar across countries. Conclusions This study suggests that patients experience perceptible benefits in physical and psychological well-being following treatment success with first-line avelumab in metastatic Merkel cell carcinoma.
\end{abstract}

\section{Introduction}

Merkel cell carcinoma (MCC) is a rare and aggressive form of skin cancer that usually appears as a single painless lump most likely on sun-exposed skin. In Europe, the incidence rate is $0.2-0.4$ cases per 100,000 person-years, whereas in the USA, it is 0.7 cases per 100,000 person-years $[1,2]$. Merkel cell carcinoma is associated with a poor prognosis, with the 5-year overall survival rate with metastatic MCC $(\mathrm{mMCC})$ estimated between 0 and $18 \%[3,4]$.

Murtuza Bharmal

murtuza.bharmal@emdserono.com

Extended author information available on the last page of the article
Surgery is the standard treatment for early-stage MCC. Historically, there have been no approved treatments for recurrent, late-stage (advanced or metastatic), non-resectable MCC and chemotherapy has been used. However, in terms of treatment effectiveness, research based on European and US registries suggests that chemotherapy in $\mathrm{mMCC}$, whether administered as first, second, or later line, was associated with only a brief tumour response, rapid emergence of chemoresistance and ultimately poor overall survival in most patients [5-7].

Avelumab is a human monoclonal antibody targeting and blocking programmed death ligand 1 . In the open-label, single-arm, registrational, multi-centre clinical trial (JAVELIN Merkel 200 trial), avelumab demonstrated a clinically meaningful and durable response for patients with mMCC and was well tolerated with few Grade 3 and no Grade 4 


\section{Key Points for Decision Makers}

This qualitative study in treatment-naïve patients with metastatic Merkel cell carcinoma showed results similar to previously published findings in patients in whom prior chemotherapy had failed.

Findings suggest that patients with treatment success with avelumab also experienced benefits in both physical and psychological well-being.

Assessing patients' experience with the disease (metastatic Merkel cell carcinoma) and with treatment (avelumab) is essential to explain how patients value the new therapy and how they feel and function while receiving the drug and complements patient-reported outcome data by providing patients perspectives.

treatment-related adverse events [8,9], and more recently as a first-line treatment $[10,11]$. In 2017, avelumab was conditionally approved by the US Food and Drug Administration to treat patients with mMCC from the age of 12 years, whereas the European Medicines Agency approved avelumab for adult patients only, making it the first available treatment option in this indication. More recently, pembrolizumab, a programmed death 1-targeted monoclonal antibody was approved for the treatment of advanced MCC in the first-line setting in the USA [12].

Besides documenting clinical effectiveness within clinical trials, in recent years, it has also become increasingly important to document treatment benefit and meaningfulness from the patient perspective to inform drug development and evaluation [13, 14]. This is particularly important in the context of rare diseases $[15,16]$. Therefore, optional semi-structured interviews were conducted with patients participating in the JAVELIN Merkel 200 trial at baseline prior to study drug administration and at weeks 13 and 25.

Findings from interviews conducted with patients with mMCC receiving avelumab as second- or laterline treatment (part A) have previously been reported [17-19]. Briefly, those patients, mostly from the USA, described MCC as painless and symptomless. Merkel cell carcinoma had a substantial psychological impact on both the patients and their relatives and friends; in most cases, patients were scared of the unknown and the future. This psychological burden whether it results from the diagnosis or from the fear about the future treatment response is common to most cancers [20-22]. However, patients with MCC, a rare and very aggressive cancer with limited treatment options, may have specific needs and expectations.
Patients with mMCC reported a clear benefit with avelumab treatment, as reflected by a substantial improved tumour status, which was associated with an improved physical and psychological status. Patient satisfaction with avelumab was high relative to their previous negative experiences with chemotherapy and radiotherapy, which patients described as highly debilitating, both physically and mentally [18].

The present article reports the findings from the qualitative interviews conducted with a large international sample of treatment-naïve patients with $\mathrm{mMCC}$ receiving avelumab as first-line treatment and aims to complement previous findings in patients with $\mathrm{mMCC}$ receiving avelumab as secondor later-line treatment [17-19].

\section{Materials and Methods}

\subsection{Study Design}

The single-arm, open-label, multicentre, international, phase II, JAVELIN Merkel 200 trial (NCT02155647) consists of two parts: part A, which enrolled 88 patients with mMCC treated with avelumab as second- or later-line treatment, and part B, which enrolled 116 patients with mMCC who were naïve to systemic therapy in the metastatic setting and were receiving avelumab as first-line treatment. Details of the design of the trial, including efficacy and safety endpoints, have been reported elsewhere $[8,11]$. The present article focuses on the part B qualitative data.

Upon recruitment, patients were invited to participate in optional qualitative interviews in all countries (Australia, France, Germany, Italy and the USA), except in Japan owing to data privacy regulations. Interviews were optional, and as part of the trial procedures, sites were not asked to document reasons for refusal.

The clinical trial protocol, including sections related to the qualitative interviews, was approved by all relevant independent ethics committees and institutional review boards and was conducted in accordance with the Declaration of Helsinki and Good Clinical Practice. Patients who agreed to be interviewed indicated their willingness to participate within the trial informed consent form.

\subsection{Patient Interviews}

Each patient who agreed to participate in the qualitative study was to be interviewed three times: one baseline interview conducted during the screening period and two follow-up interviews at weeks 13 and 25 . Interviews were conducted as 30-min audio-recorded phone conversations. The interviews were conducted by experienced qualitative interviewers who had received study-specific training on the 
approved interview guide and who were native speakers of the country of the patient being interviewed. Interviewers used a semi-structured interview guide with open-ended questions to collect spontaneously reported information from the patients. Follow-up interviews were conducted within 5 days prior to the day of the clinical visit, during which various clinical assessments were performed and results of the examinations were shared with patients. The follow-up interviews were performed in the days prior to the clinical visits to ensure the patients would report their feelings and experience without the influence of their interaction with the physicians.

For the baseline interview, open-ended questions were used to let the interviewees answer spontaneously; the probes were only used to collect in-depth knowledge if necessary. The baseline interview guide helped the interviewers to cover (1) patients' experiences from the onset of the disease to the time of diagnosis, (2) patients' experiences of the disease over the time from diagnosis to the time of the interview and (3) patients' expectations with the study treatment.

At week 13, the interviewers followed up with each of the patients using the list of concepts and sub-concepts specifically developed from the baseline qualitative analysis. The main aim was to see how individual patient's (sub)concepts had evolved since the patient started the study and received avelumab. Similarly, a list of key (sub)concepts identified and/or followed up during the interview at week 13 was established after qualitative analysis of the week 13 interview to guide the interview at week 25 . For each of the (sub) concepts, patients were reminded of certain quotes from the previous interview(s), using the following prompts: "Has this changed since our last interview?," "If yes, in which way?" and "Could you describe further?" In addition, the patients were asked if anything new (e.g. signs or symptoms, disease impact) and any turning point (e.g. occurrence of a new disease; hospitalisation) had occurred since the last interview and to describe it if anything had occurred. Then, the interviewers asked open-ended questions about the actual experience with MCC and experience within the trial while receiving avelumab.

Interviews were transcribed verbatim in local languages and translated in English. The interviewers were asked to review the transcripts to correct any misunderstandings. Any information that could identify a patient was removed from the transcripts prior to the qualitative analysis.

\subsection{Qualitative Analysis of Patient Interviews}

Interview transcripts were analysed by a trained and experienced qualitative researcher holding a Ph.D. in oncology (JL), using the Atlas.ti-V7 qualitative software package $[23,24]$. The first four interviews were coded by another researcher (IG) with experience in qualitative research. Both researchers discussed their analyses before JL continued with analysing the other interview transcripts.

The baseline interviews were analysed using thematic analysis [25]. Briefly, the researcher tagged codes to sentences of the transcripts to facilitate comprehension of the larger number of textual data. The codes were made of several levels. The first level corresponded to the general concept reported by the patients (e.g. symptoms and impacts [physical, emotional, cognitive]). The second level and sometimes third level served as descriptors to further define each symptom or impact experienced by the patient (e.g. "impact on daily life_restricted activities due to limited mobility" and "psychological well-being_not giving up/ willing to fight"). The iterative and interpretive process of constant comparison analysis was used to develop a conceptual model (i.e. the concepts, how they were grouped and the relationship between them), illustrating the journey of a patient with MCC from diagnosis to the time of the interview [26]. All concepts and sub-concepts were reviewed. Sub-concepts were merged when capturing the same underlying concept and simply adding granularity but no new key information. While in some instances frequencies of concepts are reported to complement the narratives, and provide a broad indication of the prevalence of a concept, no inferences can be drawn about the prevalence of phenomena observed beyond the sample [27, 28].

The coding of the follow-up interviews at weeks 13 and 25 was performed to capture individual patient experiences over time. For each key concept identified in the baseline or week 13 analysis and probed at week 13 or week 25 , a category was assigned that assessed changes that may have occurred between the two time points. These categories, adapted from Saldana [29], were: newly emerged, not changed/stable, improved, worsened, ceased/disappeared, missing and turning point (i.e. experience or event that may have significantly altered the perceptions and/or life course of the patient).

\section{Results}

\subsection{Patient Characteristics}

Between June 2016 and February 2018, 29 patients were interviewed during the screening period prior to receiving the first dose of avelumab (baseline interview). Overall, 19 patients had at least one follow-up interview. At week 13, 18 patients were interviewed; at week 25, 12 patients were interviewed. One patient was interviewed at baseline and week 25 only. All participants were interviewed prior to their respective clinical visit.

Of the 29 patients (mean age 72 years; $76 \%$ male) included in the baseline analysis, nine were from the USA, 
19 from Europe and one from Australia. The interviewed sample was broadly comparable to the non-interviewed sample in terms of age and sex; however, based on clinical characteristics, the interviewed sample tended to have smaller tumours at baseline and a higher proportion were fully active based on Eastern Cooperative Oncology Group performance status compared with non-interviewed patients (Table 1).

\subsection{Patient Experience with Merkel Cell Carcinoma Diagnosis}

Prior to diagnosis, patients reported having a painless "lump", "bump" or "spot." Some patients further described it as looking like a "mosquito bite", "blister" or "wart." Most patients did not report any impacts or interferences pertaining to the lump prior to their diagnosis. Few patients $(n=3)$ reported being worried or concerned because they did not know what the lump was.

When describing their journey prior to being diagnosed with MCC, several patients said that they had been misdiagnosed at first. Patients also said that their physicians referred them to various specialists and that they underwent several tests prior to being diagnosed with MCC.

Patients reported that their first reaction to the diagnosis was shock and surprise. Once the patients became aware of the seriousness of their disease, they reported being "shocked", "scared" and "worried." Four patients mentioned that their family/relatives were concerned by the diagnosis.
Patient quotes illustrating the journey of the patients from the time before diagnosis to their actual diagnosis are provided in Table 2.

\subsection{Patient Experience Living with Metastatic Merkel Cell Carcinoma}

Baseline interviews further focused on the impact of mMCC on patients' lives from diagnosis to the time of the interviews. Illustrative patient quotes are provided in Table 3. The symptoms experienced by patients with mMCC did not change much from those experienced around the time of diagnosis. Four patients specifically and spontaneously mentioned having no symptoms. One-third of the patients reported some level of pain around the tumour site, and six patients reported some swelling around the tumour site. Several patients $(n=8)$ described the progression of the disease with the occurrence of metastases or recurrence.

The majority of patients did not experience or only experienced a minor impact on their cognitive abilities and everyday lives, including their social life, body image, sleep and appetite. However, some patients mentioned experiencing limited mobility owing to the tumour location and/or due to fatigue. Two patients reported that $\mathrm{mMCC}$ prevented them from doing anything. These two patients further explained that the disease had led to poor sleep quality, interference with their social lives and restrictions in their daily activities
Table 1 Baseline demographic and clinical characteristics of interviewed and noninterviewed patients

\begin{tabular}{|c|c|c|c|}
\hline Baseline characteristics & $\begin{array}{l}\text { Patients with baseline } \\
\text { interviews }(n=29)\end{array}$ & $\begin{array}{l}\text { Patient with } \geq 1 \text { follow- } \\
\text { up interview }(n=19)\end{array}$ & $\begin{array}{l}\text { Patients without } \\
\text { baseline interviews } \\
(n=87)\end{array}$ \\
\hline Mean age (SD), years & $71.7(7.8)$ & $70.4(8.0)$ & $73.1(11.0)$ \\
\hline \multicolumn{4}{|l|}{ Sex, $n(\%)$} \\
\hline Male/female & $22(75.9) / 7(24.1)$ & $16(84.2) / 3(15.8)$ & $59(67.8) / 28(32.2)$ \\
\hline \multicolumn{4}{|l|}{ Country, $n(\%)$} \\
\hline Australia & $1(3.4)$ & $1(5.3)$ & $8(9.2)$ \\
\hline France & $7(24.1)$ & $4(21.1)$ & $29(33.3)$ \\
\hline Germany & $6(20.7)$ & $3(15.8)$ & $11(12.6)$ \\
\hline Italy & $6(20.7)$ & $5(26.3)$ & $15(17.2)$ \\
\hline USA & $9(31.0)$ & $6(31.6)$ & $20(23.0)$ \\
\hline Japan & $\mathrm{na}^{\mathrm{a}}$ & $\mathrm{na}^{\mathrm{a}}$ & $3(3.4)$ \\
\hline Spain & 0 & 0 & $1(0.9)$ \\
\hline $\begin{array}{l}\text { Mean time since initial diagno- } \\
\text { sis (SD), years }\end{array}$ & $2.2(0.8)$ & $2.4(0.8)$ & $2.3(0.8)$ \\
\hline Mean tumour size (SD), mm & $61.9(46.5)$ & $48.1(34.7)$ & $81.7(54.0)^{\mathrm{b}}$ \\
\hline \multicolumn{4}{|l|}{ ECOG performance status, $n(\%)$} \\
\hline 0 & $24(82.8)$ & $18(94.7)$ & $48(55.2)$ \\
\hline 1 & $5(17.2)$ & $1(5.3)$ & $39(44.8)$ \\
\hline
\end{tabular}

ECOG Eastern Cooperative Oncology Group, na not applicable, SD standard deviation ${ }^{a}$ No patient interviews were conducted in Japan owing to data privacy regulations

${ }^{\mathrm{b}}$ Data missing for two patients 
because of limited mobility. Focusing on physical wellbeing, some ambiguous findings were observed. While half of the patients mentioned feeling healthy and strong, the other half mentioned feeling tired, weak and fatigued.

In contrast, the psychological well-being of most patients was strongly affected. Patients mentioned being worried, scared by both future and death, and depressed. However, several patients remained positive, wanting to fight the cancer. Patients described their family and relatives as "worried" and "concerned" but also as very supportive.

The majority of patients $(n=20)$ reported that they had received radiotherapy. Some patients reported positive results from it, even if they ultimately experienced relapse. Reported side effects were quite diverse; only fatigue $(n=6)$, burns $(n=3)$ and hair loss $(n=2)$ were reported by more than one patient.

Just prior to the start of the trial, patients hoped that avelumab would let them live longer, cure the cancer or make the cancer go into remission. One-third of the patients were well informed about part A of the study and were fully aware that avelumab may not work for everyone. Figure 1 presents a conceptual model of the patients' experiences with MCC, which was developed from the concepts and sub-concepts elicited during the interviews.

\subsection{Patient Experience with Avelumab}

Among the 19 patients who had follow-up interviews, 12 reported (based on their own judgement and what they had been told during previous visits by their clinical team) that their disease had improved, four reported no changes and three reported that their disease had worsened. All three patients who reported a worsening at the week 13 interview did not complete the week 25 interview because of early study discontinuation.

Almost all patients $(n=14$ of 15$)$ responded yes to the question, "Would you recommend the study treatment (avelumab) to others with MCC?," including two patients whose disease had progressed and three patients whose disease had remained unchanged from baseline. The single patient who replied negatively to the question had stopped avelumab before the fifth dose because of side effects (pain, numbness and cramps) arising in the legs.

As shown in Fig. 2, over the course of the trial, patients with disease that improved (based on their own judgement) tended to be stable or reported some improvements in their ability to perform daily activities and in their physical well-being, including having more energy. Most patients who reported that their condition and symptoms had improved reported worrying less and being optimistic. In contrast, patients who reported that their condition had worsened tended to report a worsening of both their physical and psychological well-being.

With regard to side effects, some patients mentioned hair loss, weight loss, or changes in appetite, sleep disturbances and cramps. Nine patients reported fatigue/ tiredness on the day of and/or the day after receiving the avelumab infusion. Furthermore, some patients reported severe side effects such as heart, liver or breathing problems, which based on their interview led to treatment discontinuation.

Table 2 Selected patient quotes related to the experience of patients with Merkel cell carcinoma (MCC)

\begin{tabular}{|c|c|}
\hline Topic & Concept: patient quotes (assigned patient number, country) \\
\hline MCC, overall & $\begin{array}{l}\text { Aggressive disease: "It was clear relatively early on that it was an aggressive, fast-growing tumour." (patient 9, Ger- } \\
\text { many) } \\
\text { Rare and fatal disease: "It's a rare cancer and it's difficult to cure, as I understand it." (patient 4, USA) }\end{array}$ \\
\hline MCC, before diagnosis & $\begin{array}{l}\text { Painless: "It doesn't hurt. It's never hurt ... It doesn't hurt. When it's pressed there, there is a slight ache if you like, but } \\
\text { it's not what I would call painful." (patient 10, France) } \\
\text { Growing bump/lump: "I think the bumps on the head I thought were some kind of mosquito bite or some kind of insect } \\
\text { bite, and then when these lumps started appearing behind my ear, I chose to-you know-go to a dermatologist." } \\
\text { (patient 8, USA) } \\
\text { Diagnostic difficulties: "I live in a corner of this country, in [redacted], where they don't even know what Merkel carci- } \\
\text { noma is. I made the mistake of seeking assistance in a structure that in my opinion is totally incompetent." (patient } 28 \text {, } \\
\text { Italy) }\end{array}$ \\
\hline MCC, at diagnosis & $\begin{array}{l}\text { Psychological impact: "I was really shocked, I was really shocked and I couldn't like ... like leave it alone, I was so } \\
\text { unwell. But still, but I'd still always thought it'll get a bit better, the doctors will manage, and then it will get better, so } \\
\text { I always thought I'll manage." (patient 26, Germany) } \\
\text { Psychological impact: "I don't know ... I didn't think much, if I was always thinking of in the end, we think of death } \\
\text { more than anything else. I mean, how long will I survive? Is it going to be treated as well as the part that was in the } \\
\text { leg? Is it going to disappear or is it too developed for ... there are too many cells right and left to treat me properly, I } \\
\text { don't know." (patient 17, France) } \\
\text { Psychological impact on family: "I think for family it was kind of a shocker that one of their parents had something bad } \\
\text { going on with them." (patient 5, USA) }\end{array}$ \\
\hline
\end{tabular}


Table 3 Selected patient quotes related to symptoms and impact of metastatic Merkel cell carcinoma (mMCC) from diagnosis to the time of the interviews

\begin{tabular}{lc}
\hline Topic & Concept: patient quotes (assigned patient number, country) \\
\hline mMCC, symptoms & Symptomless disease: "Listen, physically, I don't have a problem, except for a little pain in my \\
& arm, sometimes when I move or when I make quick movements, around the cyst that I have \\
under my arm - under my left arm." (patient 17, France) \\
Fatigue/swelling in leg: "For a while now I've been feeling kind of tired, my leg feels heavy, I \\
don't ... I can't move it freely. Furthermore it's swollen, and since they began with these proce- \\
dures, it swelled up and the swelling basically never went away." (patient 20, Italy) \\
No impact on daily activities: "I can walk after all, I can do everything like before. I don't work \\
anyway, I'm retired, after all/OK/so I have no negative impacts anywhere." (patient 16, Ger- \\
many) \\
Minor impact on daily activities/fatigue: "I'm continuing with ... my activities. I have activi- \\
ties with the arrondissement town hall and for now, I can carry on performing these activities, \\
although I feel more tired than before. I have more trouble doing these activities." (patient 11, \\
France) \\
Minor impact on daily activities/slight pain: "I actually feel downright fit and healthy. Um, for a \\
few months I had very slight pressure pain in my left ... in my left shoulder, which I actually \\
wouldn't describe as pain, but a very slight pressure, which felt a bit like a muscle cramp. Um, \\
now that I know that the tumour's there, I can attribute it to that, of course. Otherwise, yeah, I \\
feel fit, uh, so I do my yoga, a bit of exercise, a bit of running, play a bit of golf, and the tumour \\
somehow doesn't impair that." (patient 12, Germany) \\
Fatigue: "Fatigue. Being tired all the time ... I don't have the energy that I had before." (patient \\
19, USA)
\end{tabular}

mMCC, impact on psychological well-being Fear of death/unknown: "And so far, no one has told me about how this disease evolves. And, um ... first of all, can it be cured? And, on the other hand, how long do I have left to live, you know. So many things, you know...that we didn't talk about and that at [redacted]. Nobody talked to me about it." (patient 11, France)

Fear of death: "I mean that's scary and frustrating because, um, it, uh, you know, the literature on Merkel cell is not very encouraging once it's metastasized. Uh, the life span is something like nine and a half months." (patient 4, USA)

Positive attitude/willing to fight: "I'm not the type of person who just ... who just slacks off. But I'm also a fighter and I said to myself ... well, let's get on with it, uh ... I've got to be positive, I know that ... I will be cured." (patient 14, France)

Support from family/relatives/friends: "All my family has been ... very supportive. The ... uh ... the family, but also, especially, all of my friends who are around me, they're all very caring, they all call me, they ... they want to help me in any possible way, in any aspect." (patient 28, Italy)

mMCC, experience with radiotherapy

Worked well, but did not ultimately prevent from recurrence: "I then had radiation. The radiation began, I think, in March and went on all through April, so basically 30 radiation sessions. And so, it was all fine until something else was discovered in late August." (patient 24, Germany)

Fatigue/burns: I haven't felt really bad through any of this ... um, the fatigue, that's the biggest thing. During the time of radiation, I was sleeping about $16 \mathrm{~h}$ a day. But I really haven't felt bad. At the end, I did get some radiation burns, and they are healed now and peeled and all that kind of stuff." (patient 19, USA)

Fatigue/infection: "Radiation was, um, was amazingly ... pretty low-key for me. I guess I felt fatigued and a little bit of skin discomfort, but it wasn't until about the last week-I had 5 weeks of it, um, a little bit over that, it was almost 6-it wasn't really until the last week or so that the skin really started to be infected." (patient 5, USA)

mMCC, expectations toward avelumab

Aware of former results/positive: "I feel fortunate that I have an avenue to at least try out this type of therapy and you know, clearly it's not working for everybody and we don't know how long it'll work or how well it'll work, but it seems to have a lot of promise and the traditional way of treating it, you know, isn't very promising." (patient 5, USA)

Hope to be cured: "If I do that, if these injections are going to cure me, uh ... Well, from what I've read, unfortunately the result is not guaranteed. Umm ... but I'm doing it because I'm hopeful that it will cure me, of course." (patient 10, France)

\section{Discussion}

This study in patient with treatment-naïve mMCC should be considered as complementary to the previously published study among chemotherapy refractory patients with mMCC [17-19].
Overall, patients reported that MCC, a rare and aggressive cancer of the skin, did not have any major impacts on their daily lives. They reported a difficult and long journey until they were correctly diagnosed with MCC. At the time of diagnosis, patients were usually not affected in general 


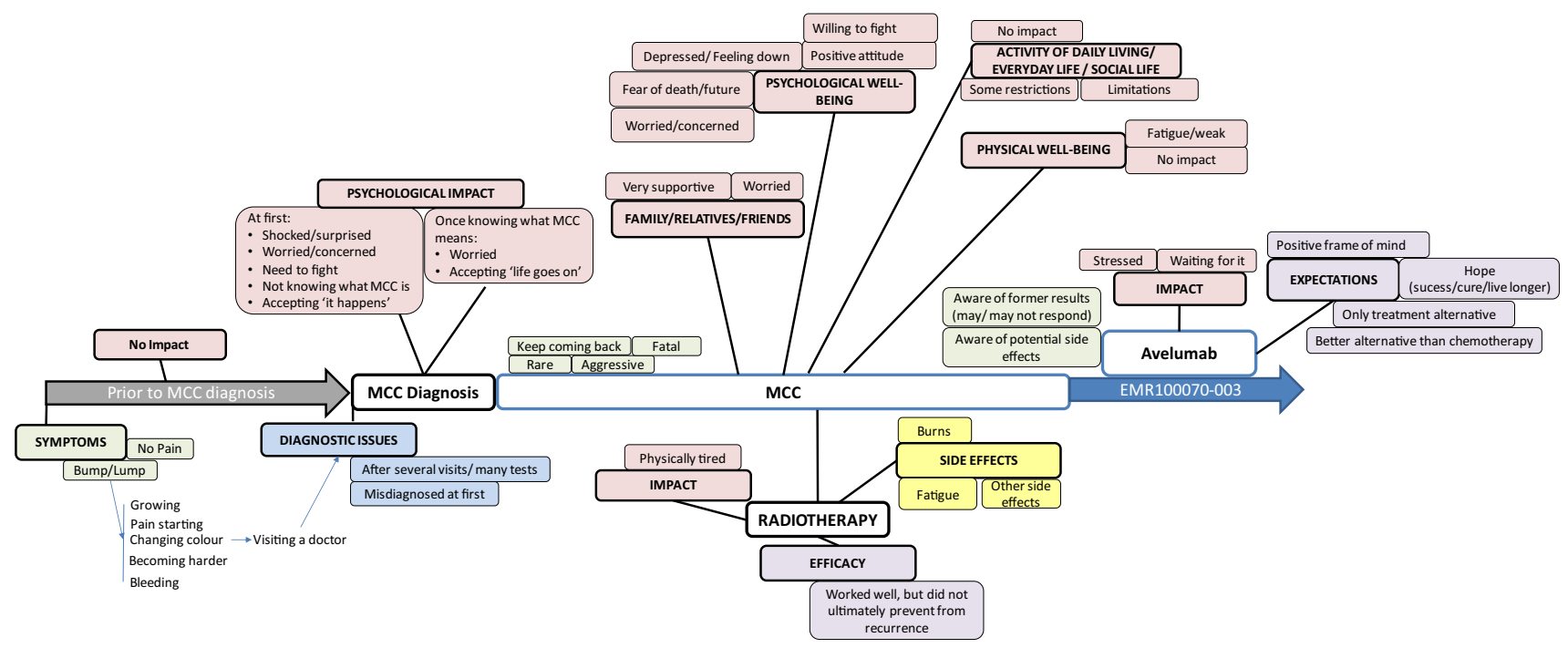

Fig. 1 Conceptual model of the journey of patients with Merkel cell carcinoma (MCC) and its management before diagnosis to study entry. Red: concepts related to an impact; yellow: concepts related to symptoms/side effects; purple: concepts related to perceived treatment efficacy and pectations; light green: concepts related to descriptive facts; light blue: concepts related to diagnostic issues

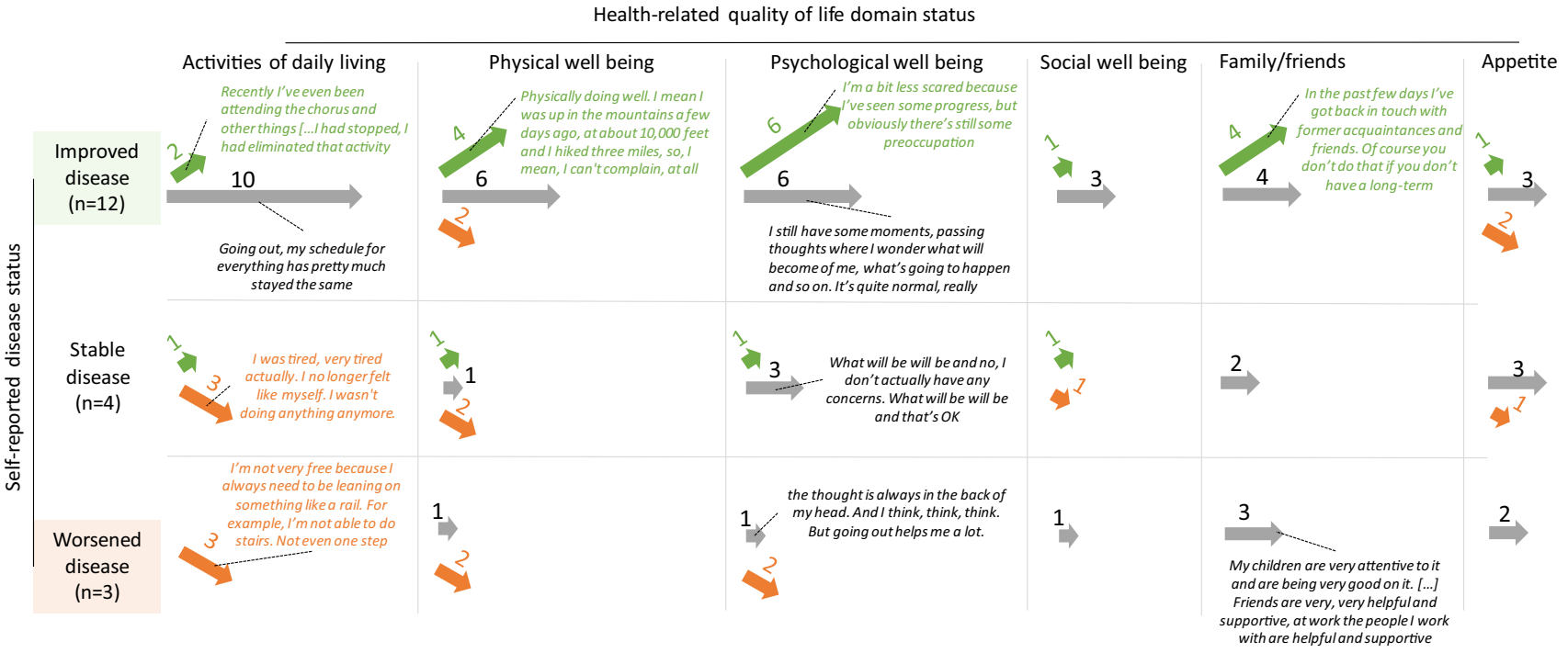

Fig. 2 Changes in various dimensions of health-related quality of life at last available time point in patients with disease that had improved $(n=12)$, were stable $(n=4)$ or had worsened $(n=3)$ per patient selfreport. Green, grey and orange arrows represent patient-reported improvement, stability and worsening in each health-related qualityof-life domain, respectively. Arrow size is proportional to the number of subjects experiencing improvement, no change and worsening in each health-related quality-of-life domain by MCC, apart from having a growing lump on their skin, for which they visited their clinician. Several patients were first misdiagnosed, and most patients had never heard about MCC until they received their diagnosis. These qualitative findings highlight that there is currently a lack of awareness around MCC from both clinical teams and patients that leads to misdiagnosis and subsequent delays in diagnosis, which in turn delays access to appropriate management. As reported in the literature, misdiagnosis and subsequent delayed diagnosis and treatment are factors leading to poor prognosis $[30,31]$. Efforts should be made to increase awareness of primary care physicians and dermatologists about MCC.

Approximately one-fourth of study participants in part B took part in the interviews [29 of $116(25 \%)$ ], similar to the participation rate in part A [19 of $88(22 \%)]$. The patient 
experiences reported here from part B of JAVELIN Merkel 200 (i.e. patients with mMCC naïve to systemic therapy in the metastatic setting) were similar to the experiences reported previously by patients in part A (i.e. patients with mMCC previously treated with chemotherapy) [19]. This could be expected considering that the psychological burden of living with MCC, and mainly anxiety towards the evolution and the impact of treatment is a central concept; first and second line may not differ in this context.

Interviews in part A were mostly conducted with US participants; there was a more diverse population (i.e. patients were from France, Germany, Italy, USA and Australia) in part $\mathrm{B}$, which may enable the generalisation of the results beyond the US population to the global population of patients with $\mathrm{mMCC}$.

In part B, although the interviewed sample was broadly comparable to the non-interviewed sample in terms of age and sex, the interviewed sample tended to have smaller tumours at baseline and a higher proportion of fully active patients based on Eastern Cooperative Oncology Group performance status than the non-interviewed sample. The depiction provided in this article may therefore not fully capture patients with the most severe experience of the condition.

Most patients reported that upon entering the trial, MCC had no major impact on their appetite, sleep or physical well-being. However, most patients were worried about their future. Over the course of the trial, after having received avelumab for several weeks, patients with disease that improved tended to be stable or reported some improvements in their ability to perform daily activities, their physical well-being and their energy level, despite not always spontaneously reporting any impairments at baseline. Most patients who reported that their condition had improved, reported worrying less and being more optimistic. In contrast, patients who reported that their condition had worsened tended to report a worsening of their physical and psychological well-being. The activities of daily living were particularly affected in those patients with a stable or worsened condition. Among the patients who reported disease improvement based on their own judgement, the psychological well-being improved in half of them. Inversely, two of the three subjects whose disease deteriorated reported a worsened psychological wellbeing. It cannot be excluded that the psychological state of an individual could have some influence on one's judgement [32]. In part A, only patients who were still responding to avelumab were interviewed. In contrast, in part B, three patients who discontinued avelumab either because they did not respond to it or they experienced side effects were also interviewed. This allowed us to consider the experiences of patients for whom avelumab was not a success. These were patients who were in good enough health to be interviewed. We are conscious that our results are probably biased by the fact that patients with less impact, and more positive feelings were more likely to respond.

Qualitative interviews with patients during clinical trials serve to document the treatment benefit and capture the meaningful effect of the drug from a patient perspective. The Food and Drug Administration is strongly committed to supporting initiatives and efforts that integrate patient input into the development program for a drug. The Food and Drug Administration has released initial guidelines on the collection and submission of patient experience data to inform medical product development and regulatory decision making [13]. The European Medicines Agency and health technology assessment bodies also acknowledge that qualitative data are important for adding information about the clinical benefit of a therapy and supporting benefit-risk balance assessments [33]. At the time of the start of this trial, there was no quantitative instruments specific to MCC, hence a melanoma-specific questionnaire, FACT-M, was administered to patients. Data from the collected qualitative interviews alongside the trial were used to support its psychometric validation [34]. A mixed-method research approach using the qualitative interview data together with the questionnaire quantitative data and clinical data to provide a wholistic perspective on patient experience has been previously published using part A data [17]. Potential future work using this part B data could be conducted applying such a mixed-method research approach to triangulate qualitative findings and quantitative findings and draw further insights on patient experience with avelumab.

\section{Conclusions}

This qualitative study in treatment-naïve patients with mMCC showed results similar to previously published findings in patients who had received avelumab as second- or later-line treatment for whom chemotherapy had failed. These findings suggest that patients experience benefits in both physical and psychological well-being following treatment success with avelumab. This qualitative approach captures information that is more in-depth and complementary to quantitative assessments documenting treatment effects.

Acknowledgements The authors thank all participating patients and their families, investigators, interviewers and trial staff.

Author contributions $\mathrm{MB}, \mathrm{AM}$ and JL contributed to the study design. JL performed the analysis, SPD, MAB, BC, NF, TG, JJG, CL, CR and JR were principal investigators in the JAVELIN Merkel 200 trial and supported recruitment of patients. All authors contributed to the data interpretation, critically reviewed the manuscript and approved the submitted version. 


\section{Compliance with Ethical Standards}

Funding This study was funded by an alliance between Merck KGaA, Darmstadt, Germany and Pfizer.

Conflict of interest Jérémy Lambert and Alexia Marrel are employees of ICON, and were paid consultancy fees by Merck KGaA. Murtuza Bharmal is an employee of EMD Serono, a business of Merck KGaA, Darmstadt, Germany. Melissa A. Burgess reports serving as a consultant or advisor for EMD Serono and Immune Design. Nicola Fazio provided speaker and advisory board services for AAA Pharma, Ipsen, Merck KGaA, Merck Sharp \& Dohme, Novartis and Pfizer. Thilo Gambichler received speakers and/or advisory board honoraria from $\mathrm{Ab}$ bvie, Almirall, Bristol-Myers Squibb, Eli Lilly, Janssen, Merck KGaA, Merck Sharp \& Dohme, Novartis, Roche, Pfizer, Pierre Fabre and Sanofi-Genzyme. Jean-Jacques Grob has received honoraria from Amgen, Bristol-Myers Squibb, Merck, Merck Sharp \& Dohme, Novartis, Pfizer, Pierre Fabre, Roche and Sanofi; reports serving as a consultant or advisor for Amgen, Brystol-Myers Squibb, Merck, Merck Sharp \& Dohme, Novartis, Pfizer, Pierre Fabre, Roche and Sanofi; is a member of a speakers' bureau for Novartis; and has received reimbursement for travel and accommodations expenses from Brystol-Myers Squibb, Merck Sharp \& Dohme, Novartis and Pierre Fabre. Céleste Lebbé has received honoraria from Amgen, Bristol-Myers Squibb, Incyte, Merck Sharp \& Dohme, Novartis, Pfizer, Pierre Fabre and Roche; reports serving as a consultant or advisor for Amgen, Bristol-Myers Squibb, Merck Sharpe \& Dohme, Novartis and Roche; is a member of a speakers' bureau for Amgen, Bristol-Myers Squibb, Novartis and Roche; has received research funding from Bristol-Myers Squibb and Roche; has received reimbursement for travel and accommodation expenses from Bristol-Myers Squibb; and has other relationships with Avantis Medical Systems. Jeffrey Russell is an employee of Immunocore. Gülseren Güzel is an employee of Merck KGaA. Sandra P. D'Angelo reports serving as a consultant or advisor for Amgen, EMD Serono, GlaxoSmithKline, Immune Design, Incyte, Merck \& Co. and Nektar; received research grants from Amgen, Bristol-Myers Squibb, Deciphera, EMD Serono, Incyte, Merck \& Co. and Nektar; and received reimbursement for travel and accommodation expenses from Adaptimmune, EMD Serono and Nektar. Caroline Robert reports advisory roles for BMS, Piere Fabre, Novartis and Amgen and reports ownership of equity from Merck KGaA, Roche, MSD, Sanofi, Biotherma and Ultimovacs. Bartosz Chmielowski has no conflict of interest to declare.

Ethics Approval The clinical trial protocol, including sections related to the qualitative interviews, was approved by all relevant independent ethics committees and institutional review boards and was conducted in accordance with the Declaration of Helsinki and Good Clinical Practice.

Consent to Participate All participants provided written informed consent prior to any study-related procedures. Patients who agreed to be interviewed indicated their willingness to participate within the informed consent form.

Data Availability For all new products or new indications approved in both the European Union and the USA after 1 January, 2014, Merck KGaA, Darmstadt, Germany will share patient-level and study-level data after deidentification, as well as redacted study protocols and clinical study reports from clinical trials in patients. These data will be shared with qualified scientific and medical researchers, upon a researcher's request, as necessary for conducting legitimate research. Such requests must be submitted in writing to the company's data sharing portal. More information can be found at https://www.merckgroup.com/en/research/ our-approach-to-research-and-development/healthcare/clinical-trials/ commitment-responsible-data-sharing.html. Where Merck KGaA has a co-research, co-development, or co-marketing/co-promotion agreement or where the product has been out-licensed, it is recognised that the responsibility for disclosure may be dependent on the agreement between parties. Under these circumstances, Merck KGaA will endeavour to gain agreement to share data in response to requests.

Open Access This article is licensed under a Creative Commons Attribution-NonCommercial 4.0 International License, which permits any non-commercial use, sharing, adaptation, distribution and reproduction in any medium or format, as long as you give appropriate credit to the original author(s) and the source, provide a link to the Creative Commons licence, and indicate if changes were made. The images or other third party material in this article are included in the article's Creative Commons licence, unless indicated otherwise in a credit line to the material. If material is not included in the article's Creative Commons licence and your intended use is not permitted by statutory regulation or exceeds the permitted use, you will need to obtain permission directly from the copyright holder. To view a copy of this licence, visit http://creativecommons.org/licenses/by-nc/4.0/.

\section{References}

1. Lebbe C, Becker JC, Grob JJ, Malvehy J, Del Marmol V, Pehamberger $\mathrm{H}$, et al. Diagnosis and treatment of Merkel cell carcinoma: European consensus-based interdisciplinary guideline. Eur J Cancer. 2015;51(16):2396-403. https://doi.org/10.1016/j. ejca.2015.06.131.

2. Paulson KG, Park SY, Vandeven NA, Lachance K, Thomas H, Chapuis AG, et al. Merkel cell carcinoma: current US incidence and projected increases based on changing demographics. J Am Acad Dermatol. 2018;78(3):457-63. https://doi.org/10.1016/j. jaad.2017.10.028(e2).

3. Allen PJ, Bowne WB, Jaques DP, Brennan MF, Busam K, Coit DG. Merkel cell carcinoma: prognosis and treatment of patients from a single institution. J Clin Oncol. 2005;23(10):2300-9. https ://doi.org/10.1200/jco.2005.02.329.

4. Santamaria-Barria JA, Boland GM, Yeap BY, Nardi V, Dias-Santagata D, Cusack JC Jr. Merkel cell carcinoma: 30-year experience from a single institution. Ann Surg Oncol. 2013;20(4):1365-73. https://doi.org/10.1245/s10434-012-2779-3.

5. Becker JC, Lorenz E, Ugurel S, Eigentler TK, Kiecker F, Pfohler $\mathrm{C}$, et al. Evaluation of real-world treatment outcomes in patients with distant metastatic Merkel cell carcinoma following secondline chemotherapy in Europe. Oncotarget. 2017;8(45):79731-41. https://doi.org/10.18632/oncotarget.19218.

6. Cowey CL, Mahnke L, Espirito J, Helwig C, Oksen D, Bharmal M. Real-world treatment outcomes in patients with metastatic Merkel cell carcinoma treated with chemotherapy in the USA. Future Oncol. 2017;13(19):1699-710. https://doi.org/10.2217/ fon-2017-0187.

7. Iyer JG, Blom A, Doumani R, Lewis C, Tarabadkar ES, Anderson $\mathrm{A}$, et al. Response rates and durability of chemotherapy among 62 patients with metastatic Merkel cell carcinoma. Cancer Med. 2016;5(9):2294-301. https://doi.org/10.1002/cam4.815.

8. Kaufman HL, Russell J, Hamid O, Bhatia S, Terheyden P, D'Angelo SP, et al. Avelumab in patients with chemotherapyrefractory metastatic Merkel cell carcinoma: a multicentre, singlegroup, open-label, phase 2 trial. Lancet Oncol. 2016;17(10):137485. https://doi.org/10.1016/s1470-2045(16)30364-3.

9. Kaufman HL, Russell JS, Hamid O, Bhatia S, Terheyden P, D'Angelo SP, et al. Updated efficacy of avelumab in patients with previously treated metastatic Merkel cell carcinoma after $\geq 1$ year of follow-up: JAVELIN Merkel 200, a phase 2 clinical trial. J 
Immunother Cancer. 2018;6(1):7. https://doi.org/10.1186/s4042 5-017-0310-x.

10. D'Angelo SP, Hunger M, Brohl AS, Nghiem P, Bhatia S, Hamid $\mathrm{O}$, et al. Early objective response to avelumab treatment is associated with improved overall survival in patients with metastatic Merkel cell carcinoma. Cancer Immunol Immunother. 2019;68(4):609-18. https://doi.org/10.1007/s00262-018-02295-4.

11. D'Angelo SP, Russell J, Lebbe C, Chmielowski B, Gambichler $\mathrm{T}$, Grob JJ, et al. Efficacy and safety of first-line avelumab treatment in patients with stage IV metastatic Merkel cell carcinoma: a preplanned interim analysis of a clinical trial. JAMA Oncol. 2018;4(9):e180077. https://doi.org/10.1001/jamaoncol.2018.0077.

12. Nghiem PT, Bhatia S, Lipson EJ, Kudchadkar RR, Miller NJ, Annamalai L, et al. PD-1 blockade with pembrolizumab in advanced Merkel-cell carcinoma. N Engl J Med. 2016;374(26):2542-52. https://doi.org/10.1056/NEJMoa1603702.

13. Barlas S. FDA to collect patient experience data: upcoming guidance documents will spell out what and how to submit. Pharm Ther. 2018;43(6):318-36.

14. Chalasani M, Vaidya P, Mullin T. Enhancing the incorporation of the patient's voice in drug development and evaluation. Res Involv Engagem. 2018;4:10. https://doi.org/10.1186/s40900-018-0093-3.

15. Britten N. Qualitative research on health communication: what can it contribute? Patient Educ Couns. 2011;82(3):384-8. https ://doi.org/10.1016/j.pec.2010.12.021.

16. O'Cathain A, Thomas KJ, Drabble SJ, Rudolph A, Hewison J. What can qualitative research do for randomised controlled trials? A systematic mapping review. BMJ Open. 2013;3(6):e002889. https://doi.org/10.1136/bmjopen-2013-002889.

17. Bharmal M, Guillemin I, Marrel A, Arnould B, Lambert J, Hennessy $\mathrm{M}$, et al. How to address the challenges of evaluating treatment benefits-risks in rare diseases? A convergent mixed methods approach applied within a Merkel cell carcinoma phase II clinical trial. Orphanet J Rare Dis. 2018;13(1):95.

18. Bharmal M, Marrel A, Hennessy M, Fofana F, Lambert J, Arnould B. Comparative effectiveness of avelumab versus chemotherapy in Merkel cell carcinoma: innovative use of patient insights. J Comp Eff Res. 2018;7(9):881-90. https://doi.org/10.2217/ cer-2018-0048.

19. Kaufman HL, Dias Barbosa C, Guillemin I, Lambert J, Mahnke L, Bharmal M. Living with Merkel cell carcinoma (MCC): development of a conceptual model of MCC based on patient experiences. Patient. 2018;11(4):439-49. https://doi.org/10.1007/s4027 1-018-0301-0.

20. Al-Shakhli H, Harcourt D, Kenealy J. Psychological distress surrounding diagnosis of malignant and nonmalignant skin lesions at a pigmented lesion clinic. J Plast Reconstr Aesthet Surg. 2006;59(5):479-86. https://doi.org/10.1016/j.bjps.2005.01.010.
21. Burdon-Jones D, Thomas P, Baker R. Quality of life issues in nonmetastatic skin cancer. Br J Dermatol. 2010;162(1):147-51. https://doi.org/10.1111/j.1365-2133.2009.09469.x.

22. Cinar D, Yildirim Y, Yesilbalkan AU, Pamuk A. Experiences of cancer patients: a qualitative study. Int J Caring Sci. 2018;11(3):1456-66.

23. Friese S, Ringmayr TG. ATLAS.ti. 7th ed. Berlin: Scientific Software Development; 2013.

24. Silver C, Lewins A. Using software in qualitative research: a stepby-step guide. 2nd ed. London: SAGE publications ltd; 2014. https ://doi.org/10.4135/9781473906907.

25. Charmaz K. Grounded theory in the 21st century. 3rd ed. Thousand Oaks: Sage; 2005.

26. US Food and Drug Administration. Guidance for industry: patientreported outcome measures: use in medical product development to support labeling claims. 2009. https://www.fda.gov/downloads/ Drugs/GuidanceComplianceRegulatoryInformation/Guidances/ UCM193282.pdf. Accessed 7 Oct 2019.

27. Neale J, Miller P, West R. Reporting quantitative information in qualitative research: guidance for authors and reviewers. Addiction. 2014;109(2):175-6. https://doi.org/10.1111/add.12408.

28. Sandelowski M. Real qualitative researchers do not count: the use of numbers in qualitative research. Res Nurs Health. 2001;24(3):230-40. https://doi.org/10.1002/nur.1025.

29. Saldana J. The coding manual for qualitative researchers. Thousand Oaks: Sage; 2012.

30. Rebecca AM, Craft RO, Smith AA. Digital Merkel cell carcinoma. Can J Plast Surg. 2005;13(4):199-202. https://doi. org/10.1177/229255030501300407.

31. Tsang G, O'Brien P, Robertson R, Hamilton C, Wratten C, Denham J. All delays before radiotherapy risk progression of Merkel cell carcinoma. Australas Radiol. 2004;48(3):371-5. https://doi. org/10.1111/j.0004-8461.2004.01321.x.

32. Siegel E, Wormwood J, Quigley K, Barrett L. Seeing what you feel: affect drives visual perception of structurally neutral faces. Version 2. Psychol Sci. 2018;29(4):496-503. https://doi. org/10.1177/0956797617741718.

33. Gnanasakthy A, DeMuro C, Mordin M, Copley-Merriman K, Mauskopf J. The role of the patient voice in health technology assessment. Value Health. 2010;13(3):A19. https://doi. org/10.1016/S1098-3015(10)72074-X.

34. Bharmal M, Fofana F, Barbosa CD, Williams P, Mahnke L, Marrel A, et al. Psychometric properties of the FACT-M questionnaire in patients with Merkel cell carcinoma. Health Qual Life Outcomes. 2017;15(1):247. https://doi.org/10.1186/s12955-017-0815-5. 


\section{Affiliations}

Jérémy Lambert ${ }^{1}$ - Alexia Marrel ${ }^{1}$ - Sandra P. D'Angelo ${ }^{2} \cdot$ Melissa A. Burgess $^{3} \cdot$ Bartosz Chmielowski $^{4} \cdot$ Nicola Fazio $^{5}$. Thilo Gambichler ${ }^{6}$. Jean-Jacques Grob ${ }^{7}$. Céleste Lebbé ${ }^{8}$. Caroline Robert ${ }^{9}$. Jeffrey Russell ${ }^{10}$. Gülseren Güzel ${ }^{11}$. Murtuza Bharmal ${ }^{12}$

1 Patient-Centred Outcomes, ICON Plc, Lyon, France

2 University of Pittsburgh Medical Center Hillman Cancer Center, Pittsburgh, PA, USA

3 Memorial Sloan Kettering Cancer Center, New York, NY, USA

4 UCLA Jonsson Comprehensive Cancer Center, Los Angeles, CA, USA

5 Division of Gastrointestinal Medical Oncology and Neuroendocrine Tumours, European Institute of Oncology (IEO), IRCCS, Milan, Italy

6 Skin Cancer Center, Department of Dermatology, Ruhr University, Bochum, Germany
7 Department of Dermatology, Aix-Marseille University, Marseille, France

8 Université de Paris, INSERM U976, and Dermatology and CIC, AP-HP, Saint Louis Hospital, Paris, France

9 Department of Dermatology, Institut Gustave Roussy, Villejuif, France

10 Immunocore, LLC, Conshohocken, PA, USA

11 Merck KGaA, Darmstadt, Germany

12 EMD Serono, One Technology Place, Rockland, MA 02370, USA 